International Journal of Pure and Applied Mathematics

Volume 96 No. 3 2014, 377-390

ISSN: $1311-8080$ (printed version); ISSN: 1314-3395 (on-line version)

url: http://www.ijpam.eu

doi: http://dx.doi.org/10.12732/ijpam.v96i3.8

ijpam.eu

\title{
VARIOUS OPERATIONS AND FUZZY PREORDERS INDUCED BY ALEXANDROV TOPOLOGIES
}

\author{
Yong Chan Kim \\ Department of Mathematics \\ Gangneung-Wonju University \\ Gangneung, Gangwondo, 210-702, KOREA
}

\begin{abstract}
In this paper, we investigate the properties of various operations and fuzzy preorders induced by Alexandrov topologies in complete residuated lattices. Moreover, we give their examples.
\end{abstract}

AMS Subject Classification: 03E72, 06A15, 06F07, 54F05

Key Words: complete residuated lattices, fuzzy preorders, Alexandrov topologies

\section{Introduction}

Pawlak $[8,9]$ introduced rough set theory as a formal tool to deal with imprecision and uncertainty in data analysis. Hájek [2] introduced a complete residuated lattice which is an algebraic structure for many valued logic. Bělohlávek [1] investigated information systems and decision rules in complete residuated lattices.

On the other hand, Kortelainen [6] investigated the relation between topologies and rough sets. The relationships between Alexandrov topologies and fuzzy rough sets are studied $[3-5,7,10]$. Algebraic structures of fuzzy rough sets are developed in many directions [10-12].

Received: July 16, 2014

(c) 2014 Academic Publications, Ltd. url: www.acadpubl.eu 
In this paper, we investigate the properties of various operations and fuzzy preorders induced by Alexandrov topologies in complete residuated lattices. Moreover, we give their examples.

\section{Preliminaries}

Definition 2.1. $[1,2]$ An algebra $(L, \wedge, \vee, \odot, \rightarrow, \perp, \top)$ is called a complete residuated lattice if it satisfies the following conditions:

(C1) $L=(L, \leq, \vee, \wedge, \perp, \top)$ is a complete lattice with the greatest element $\top$ and the least element $\perp$;

(C2) $(L, \odot, \top)$ is a commutative monoid;

(C3) $x \odot y \leq z$ iff $x \leq y \rightarrow z$ for $x, y, z \in L$.

In this paper, we assume $\left(L, \wedge, \vee, \odot, \rightarrow{ }^{*} \perp, \top\right)$ is a complete residuated lattice with a strong negation;i.e. $x^{* *}=x$. For $\alpha \in L, A, \top_{x} \in L^{X},(\alpha \rightarrow A)(x)=$ $\alpha \rightarrow A(x), \quad(\alpha \odot A)(x)=\alpha \odot A(x)$ and $\top_{x}(x)=\top, \top_{x}(x)=\perp$, otherwise.

Lemma 2.2. $[1,2]$ For each $x, y, z, x_{i}, y_{i} \in L$, the following properties hold.

(1) If $y \leq z$, then $x \odot y \leq x \odot z$.

(2) If $y \leq z$, then $x \rightarrow y \leq x \rightarrow z$ and $z \rightarrow x \leq y \rightarrow x$.

(3) $x \rightarrow y=\top$ iff $x \leq y$.

(4) $x \odot\left(\bigvee_{i \in \Gamma} y_{i}\right)=\bigvee_{i \in \Gamma}\left(x \odot y_{i}\right)$.

(5) $x \rightarrow\left(\bigwedge_{i \in \Gamma} y_{i}\right)=\bigwedge_{i \in \Gamma}\left(x \rightarrow y_{i}\right)$ and $\left(\bigvee_{i \in \Gamma} x_{i}\right) \rightarrow y=\bigwedge_{i \in \Gamma}\left(x_{i} \rightarrow y\right)$.

(6) $\bigvee_{i \in \Gamma} x_{i} \rightarrow \bigvee_{i \in \Gamma} y_{i} \geq \bigwedge_{i \in \Gamma}\left(x_{i} \rightarrow y_{i}\right)$ and $\bigwedge_{i \in \Gamma} x_{i} \rightarrow \bigwedge_{i \in \Gamma} y_{i} \geq \bigwedge_{i \in \Gamma}\left(x_{i} \rightarrow\right.$ $\left.y_{i}\right)$.

(7) $(x \rightarrow y) \odot x \leq y$ and $(y \rightarrow z) \odot(x \rightarrow y) \leq(x \rightarrow z)$.

(8) $x \rightarrow y \leq(y \rightarrow z) \rightarrow(x \rightarrow z)$ and $x \rightarrow y \leq(z \rightarrow x) \rightarrow(z \rightarrow y)$.

(9) $\bigwedge_{i \in \Gamma} x_{i}^{*}=\left(\bigvee_{i \in \Gamma} x_{i}\right)^{*}$ and $\bigvee_{i \in \Gamma} x_{i}^{*}=\left(\bigwedge_{i \in \Gamma} x_{i}\right)^{*}$.

(10) $(x \odot y) \rightarrow z=x \rightarrow(y \rightarrow z)=y \rightarrow(x \rightarrow z)$ and $(x \odot y)^{*}=x \rightarrow y^{*}$.

(11) $x^{*} \rightarrow y^{*}=y \rightarrow x$ and $(x \rightarrow y)^{*}=x \odot y^{*}$.

(12) $y \rightarrow z \leq x \odot y \rightarrow x \odot z$.

(13) $x \rightarrow y \odot z \geq(x \rightarrow y) \odot z$ and $(x \rightarrow y) \rightarrow z \geq x \odot(y \rightarrow z)$.

Definition 2.3. $[4,5,7]$ A subset $\tau \subset L^{X}$ is called an Alexandrov topology if it satisfies:

(T1) $\perp_{X}, \top_{X} \in \tau$ where $\top_{X}(x)=\top$ and $\perp_{X}(x)=\perp$ for $x \in X$.

(T2) If $A_{i} \in \tau$ for $i \in \Gamma, \bigvee_{i \in \Gamma} A_{i}, \bigwedge_{i \in \Gamma} A_{i} \in \tau$.

(T3) $\alpha \odot A \in \tau$ for all $\alpha \in L$ and $A \in \tau$.

(T4) $\alpha \rightarrow A \in \tau$ for all $\alpha \in L$ and $A \in \tau$. 
Definition 2.4. $[1,3,7]$ Let $X$ be a set. A function $e_{X}: X \times X \rightarrow L$ is called:

(E1) reflexive if $e_{X}(x, x)=\top$ for all $x \in X$,

(E2) transitive if $e_{X}(x, y) \odot e_{X}(y, z) \leq e_{X}(x, z)$, for all $x, y, z \in X$,

(E3) anti-symmetric if $e_{X}(x, y)=e_{X}(y, x)=\top$, then $x=y$.

If $e$ satisfies (E1) and (E2), $\left(X, e_{X}\right)$ is a fuzzy preordered set. If $e$ satisfies (E1), (E2) and (E3), $\left(X, e_{X}\right)$ is a fuzzy partially order set.

Example 2.5. (1) We define a function $e_{L}: L \times L \rightarrow L$ as $e_{L}(x, y)=x \rightarrow y$. Then $\left(L, e_{L}\right)$ is a fuzzy partially order set.

(2) We define a function $e_{L^{X}}: L^{X} \times L^{X} \rightarrow L$ as $e_{L^{X}}(A, B)=\bigwedge_{x \in X}(A(x) \rightarrow$ $B(x))$. Then $\left(L^{X}, e_{L^{X}}\right)$ is a fuzzy partially order set from Lemma $1.2(7)$.

Definition 2.6. $[11,12]$ Let $\left(X, e_{X}\right)$ be a fuzzy partially ordered set and $A \in L^{X}$.

(1) A point $x_{0}$ is called a join of $A$, denoted by $x_{0}=\sqcup A$, if it satisfies

(J1) $A(x) \leq e_{X}\left(x, x_{0}\right)$,

$(\mathrm{J} 2) \bigwedge_{x \in X}\left(A(x) \rightarrow e_{X}(x, y)\right) \leq e_{X}\left(x_{0}, y\right)$.

A point $x_{1}$ is called a meet of $A$, denoted by $x_{1}=\sqcap A$, if it satisfies

(M1) $A(x) \leq e_{X}\left(x_{1}, x\right)$,

(M2) $\bigwedge_{x \in X}\left(A(x) \rightarrow e_{X}(y, x)\right) \leq e_{X}\left(y, x_{1}\right)$.

Remark 2.7. [11,12] Let $\left(X, e_{X}\right)$ be a fuzzy partially ordered set and $A \in L^{X}$.

(1) $x_{0}$ is a join of $A$ iff $\bigwedge_{x \in X}\left(A(x) \rightarrow e_{X}(x, y)\right)=e_{X}\left(x_{0}, y\right)$.

(2) $x_{1}$ is a meet of $A$ iff $\bigwedge_{x \in X}\left(A(x) \rightarrow e_{X}(y, x)\right)=e_{X}\left(y, x_{1}\right)$.

(3) If $x_{0}$ is a join of $A$, then it is unique because $e_{X}\left(x_{0}, y\right)=e_{X}\left(y_{0}, y\right)$ for all $y \in X$, put $y=x_{0}$ or $y=y_{0}$, then $e_{X}\left(x_{0}, y_{0}\right)=e_{X}\left(y_{0}, x_{0}\right)=\top$ implies $x_{0}=y_{0}$. Similarly, if a meet of $A$ exist, then it is unique.

Remark 2.8. [11,12] Let $\left(L^{X}, e_{L^{X}}\right)$ be a fuzzy partially ordered set and $\Phi \in L^{L^{X}}$.

(1) If $e_{L^{X}}(A, B)=e_{L^{X}}(C, B)$ for all $B \in L^{X}$, for $B=\top_{X}^{*}, A=C$.

(2) If $e_{L^{X}}(A, B)=e_{L^{X}}(A, C)$ for all $B \in L^{X}$, for $A=\top_{X}^{X}, B=C$.

(3) Since $\bigwedge_{A \in L^{X}}\left(\Phi(A) \rightarrow e_{L^{X}}(A, B)\right)=e_{L^{X}}\left(\bigvee_{A \in L^{X}}(\Phi(A) \odot A), B\right)=$ $e_{L^{X}}(\sqcup \Phi, B)$, then $\sqcup \Phi=\bigvee_{A \in L^{X}}(\Phi(A) \odot A)$.

(4) Since $\bigwedge_{A \in L^{X}}\left(\Phi(A) \rightarrow e_{L^{X}}(B, A)=\bigwedge_{A \in L^{X}} e_{L^{X}}(B,(\Phi(A) \rightarrow A))=\right.$ $e_{L^{X}}\left(B, \bigwedge_{A \in L^{X}}(\Phi(A) \rightarrow A)\right)$, then $\sqcap \Phi=\bigwedge_{A \in L^{X}}(\Phi(A) \rightarrow A)$.

Theorem 2.9. [4] A structure $\tau$ is an Alexandrov topology on $X$ iff $\tau^{*}=$ $\left\{A^{*} \in L^{X} \mid A \in \tau\right\}$ is an Alexandrov topology on $X$. 


\section{Various Operations and Fuzzy Preorders Induced by Alexandrov Topologies}

Theorem 3.1. Let $\tau$ be an Alexandrov topology on $X$. Define $\mathcal{K}_{\tau}: L^{X} \rightarrow L^{X}$ as follows:

$$
\mathcal{K}_{\tau}(A)=\bigvee_{B \in \tau}\left(e_{L^{X}}\left(B, A^{*}\right) \odot B\right) .
$$

Then the following properties hold.

(1) $e_{L^{X}}(A, B) \leq e_{L^{X}}\left(\mathcal{K}_{\tau}(B), \mathcal{K}_{\tau}(A)\right)$, for all $A, B \in L^{X}$.

(2) $\mathcal{K}_{\tau}(A) \leq A^{*}$ for all $A \in L^{X}$.

(3) $\mathcal{K}_{\tau}\left(\mathcal{K}_{\tau}^{*}(A)\right)=\mathcal{K}_{\tau}(A)$ for all $A \in L^{X}$.

(4) $\mathcal{K}_{\tau}(\alpha \odot A)=\alpha \rightarrow \mathcal{K}_{\tau}(A)$ for all $\alpha \in L, A \in L^{X}$.

(5) $\mathcal{K}_{\tau}\left(\bigvee_{i \in \Gamma} A_{i}\right)=\bigwedge_{i \in \Gamma} \mathcal{K}_{\tau}\left(A_{i}\right)$ for all $A_{i} \in L^{X}$.

(6) $\mathcal{K}_{\tau}(\sqcup \Phi)=\sqcap \mathcal{K}_{\tau}(\Phi)$ for each $\Phi: L^{X} \rightarrow L$ where $\mathcal{K}_{\tau} \rightarrow L^{L^{X}} \rightarrow L^{L^{X}}$ defined as $\mathcal{K}_{\tau}(\Phi)(B)=\bigvee_{\mathcal{K}_{\tau}(A)=B} \Phi(A)$.

(7) $\mathcal{K}_{\tau}(A)=\bigvee\left\{B \in L^{X} \mid B \leq A^{*}, B \in \tau\right\}$.

(8) Define $\tau_{\mathcal{K}_{\tau}}=\left\{A \mid A^{*}=\mathcal{K}_{\tau}(A)\right\}=\left\{\mathcal{K}_{\tau}^{*}(A) \mid A \in L^{X}\right\}$. Then $\tau=\tau_{\mathcal{K}_{\tau}}^{*}$.

(9) Define $e_{\tau}: X \times X \rightarrow L$ as

$$
e_{\tau}(x, y)=\bigwedge_{A \in \tau}(A(x) \rightarrow A(y))
$$

Then $e_{\tau}$ is a fuzzy preorder such that

$$
e_{\tau}(x, y)=\bigwedge_{z \in X}\left(\mathcal{K}_{\tau}\left(\top_{z}\right)(x) \rightarrow \mathcal{K}_{\tau}\left(\top_{z}\right)(y) .\right.
$$

(10) There exists a fuzzy preorder $e_{K_{\tau}}: X \times X \rightarrow L$ such that

$$
\mathcal{K}_{\tau}(A)(y)=\bigwedge_{x \in X}\left(e_{K_{\tau}}(x, y) \rightarrow A^{*}(x)\right) .
$$

Moreover, $e_{K_{\tau}}=e_{\tau}^{-1}$.

Proof. (1) By Lemma 2.2 (6,8,12), we have

$$
\begin{aligned}
& e_{L^{X}}\left(\mathcal{K}_{\tau}(B), \mathcal{K}_{\tau}(A)\right) \\
& =\bigwedge_{x \in X}\left(\bigvee_{C \in \tau}\left(e_{L^{X}}\left(C, B^{*}\right) \odot C(x)\right) \rightarrow \bigvee_{D \in \tau}\left(e_{L^{X}}\left(D, A^{*}\right) \odot D(x)\right)\right. \\
& \geq \bigwedge_{x \in X} \bigwedge_{C \in \tau}\left(\left(e_{L^{X}}\left(C, B^{*}\right) \odot C(x)\right) \rightarrow\left(e_{L^{X}}\left(C, A^{*}\right) \odot C(x)\right)\right. \\
& \geq \bigwedge_{C \in \tau}\left(\left(e_{L^{X}}\left(C, B^{*}\right) \rightarrow\left(e_{L^{X}}\left(C, A^{*}\right)\right)\right.\right. \\
& \geq e_{L^{X}}\left(B^{*}, A^{*}\right)=e_{L^{X}}(A, B)
\end{aligned}
$$


(2) Since $e_{L^{X}}\left(C, A^{*}\right) \odot C \leq A^{*}$ from Lemma $2.2(7), \mathcal{K}_{\tau}(A) \leq A^{*}$.

(3) Since $\mathcal{K}_{\tau}(A) \in \tau$, then $\mathcal{K}_{\tau}\left(\mathcal{K}_{\tau}^{*}(A)\right) \geq e_{L^{X}}\left(\mathcal{K}_{\tau}(A), \mathcal{K}_{\tau}(A)\right) \odot \mathcal{K}_{\tau}(A)=$ $\mathcal{K}_{\tau}(A)$. By $(2), \mathcal{K}_{\tau}\left(\mathcal{K}_{\tau}^{*}(A)\right)=\mathcal{K}_{\tau}(A)$.

(4) Since $\alpha \rightarrow \mathcal{K}_{\tau}(A) \leq \alpha \rightarrow A^{*}=(\alpha \odot A)^{*}$ and $\alpha \rightarrow \mathcal{K}_{\tau}(A) \in \tau$,

$$
\begin{aligned}
\mathcal{K}_{\tau}(\alpha \odot A) & \geq e_{L^{X}}\left(\alpha \rightarrow \mathcal{K}_{\tau}(A),(\alpha \odot A)^{*}\right) \odot\left(\alpha \rightarrow \mathcal{K}_{\tau}(A)\right) \\
& =\alpha \rightarrow \mathcal{K}_{\tau}(A), \\
\mathcal{K}_{\tau}(\alpha \odot A)= & \bigvee_{B \in \tau}\left(e_{L^{X}}\left(B, \alpha \rightarrow A^{*}\right) \odot B\right) \\
= & \bigvee_{B \in \tau}\left(\left(\alpha \rightarrow e_{L^{X}}\left(B, A^{*}\right)\right) \odot B\right) \\
\leq & \left.\alpha \rightarrow \bigvee_{B \in \tau}\left(e_{L^{X}}\left(B, A^{*}\right)\right) \odot B\right)(\text { by Lemma } 2.2(13)) \\
= & \alpha \rightarrow \mathcal{K}_{\tau}(A) .
\end{aligned}
$$

Thus, $\mathcal{K}_{\tau}(\alpha \odot A)==\alpha \rightarrow \mathcal{K}_{\tau}(A)$.

(5) By (1), since $\mathcal{K}_{\tau}(B) \leq \mathcal{K}_{\tau}(A)$ for $A \leq B, \mathcal{K}_{\tau}\left(\bigvee_{i \in \Gamma} A_{i}\right) \leq \bigwedge_{i \in \Gamma} \mathcal{K}_{\tau}\left(A_{i}\right)$. Since $\bigwedge_{i \in \Gamma} \mathcal{K}_{\tau}\left(A_{i}\right) \leq \bigwedge_{i \in \Gamma} A_{i}^{*}$ and $\bigwedge_{i \in \Gamma} \mathcal{K}_{\tau}\left(A_{i}\right) \in \tau$, we have

$$
\begin{aligned}
\mathcal{K}_{\tau}\left(\bigvee_{i \in \Gamma} A_{i}\right) & \geq e_{L^{X}}\left(\bigwedge_{i \in \Gamma} \mathcal{K}_{\tau}\left(A_{i}\right), \bigwedge_{i \in \Gamma} A_{i}^{*}\right) \odot \bigwedge_{i \in \Gamma} \mathcal{K}_{\tau}\left(A_{i}\right) \\
& =\bigwedge_{i \in \Gamma} \mathcal{K}_{\tau}\left(A_{i}\right) .
\end{aligned}
$$

Hence $\mathcal{K}_{\tau}\left(\bigvee_{i \in \Gamma} A_{i}\right)==\bigwedge_{i \in \Gamma} \mathcal{K}_{\tau}\left(A_{i}\right)$.

(6) For each $\Phi: L^{X} \rightarrow L$, put $Q=\sqcap \mathcal{K}_{\tau}(\Phi)$. Since $\mathcal{K}_{\tau} \rightarrow(\Phi): L^{X} \rightarrow L$ is a map, we have $\sqcap \mathcal{K}_{\tau}^{\rightarrow}(\Phi)=\bigwedge_{C \in L^{X}}\left(\mathcal{K}_{\tau}(\Phi)(C) \rightarrow C\right)$ and $Q=\sqcap \mathcal{K}_{\tau}(\Phi)=$ $\mathcal{K}_{\tau}(\sqcup \Phi)$ from:

$$
\begin{aligned}
e_{L^{X}}(B, Q) & =\bigwedge_{C \in L^{X}}\left(\mathcal{K}_{\tau} \rightarrow(\Phi)(C) \rightarrow e_{L^{X}}(B, C)\right. \\
& =e_{L^{X}}\left(B, \bigwedge_{C \in L^{X}}\left(\mathcal{K}_{\tau} \rightarrow(\Phi)(C) \rightarrow C\right)\right) \\
& =e_{L^{X}}\left(B, \bigwedge_{C \in L^{X}}\left(\bigvee_{\mathcal{K}_{\tau}(A)=C} \Phi(A) \rightarrow C\right)\right) \\
& =e_{L^{X}}\left(B, \bigwedge_{A \in L^{X}}\left(\Phi(A) \rightarrow \mathcal{K}_{\tau}(A)\right)\right) \\
& =e_{L^{X}}\left(B, \mathcal{K}_{\tau}\left(\bigvee_{A \in L^{X}}(\Phi(A) \odot A)\right)\right) \text { (by (4) and (5)) } \\
& =e_{L^{X}}\left(B, \mathcal{K}_{\tau}(\sqcup \Phi)\right) .
\end{aligned}
$$

(7) Put $K(A)=\bigvee\left\{B \in L^{X} \mid B \leq A^{*}, B \in \tau\right\}$. Since $K(A) \leq A^{*}$ and $K(A) \in \tau$, we have

$$
\mathcal{K}_{\tau}(A)=\bigvee_{B \in \tau}\left(e_{L^{X}}\left(B, A^{*}\right) \odot B\right) \geq e_{L^{X}}\left(K(A), A^{*}\right) \odot K(A)=K(A) .
$$

Since $\mathcal{K}_{\tau}(A) \leq A$ and $\mathcal{K}_{\tau}(A) \in \tau$, we have $K(A) \geq \mathcal{K}_{\tau}(A)$. Hence $\mathcal{K}_{\tau}=K$.

(8) Put $\tau_{1}=\left\{\mathcal{K}_{\tau}^{*}(A) \mid A \in L^{X}\right\}$. Let $A \in \tau_{\mathcal{K}_{\tau}}$. Then $A=\mathcal{K}_{\tau}^{*}(A) \in \tau_{1}$. Let $\mathcal{K}_{\tau}^{*}(A) \in \tau_{1}$. By $(3), \mathcal{K}_{\tau}\left(\mathcal{K}_{\tau}^{*}(A)\right)=\mathcal{K}_{\tau}(A)$. So, $\mathcal{K}_{\tau}^{*}(A) \in \tau_{\mathcal{K}_{\tau}}$. Thus $\tau_{\mathcal{K}_{\tau}}=\tau_{1}$. 
Moreover, $\tau=\tau_{\mathcal{K}_{\tau}}^{*}$ from:

$$
A \in \tau \text { iff } \mathcal{K}_{\tau}\left(A^{*}\right)=A \text { iff } A \in \tau_{\mathcal{K}_{\tau}}^{*}
$$

(9) Since $A=\bigvee_{x \in X}\left(A(x) \odot \top_{x}\right)$, by (4) and (5), $\mathcal{K}_{\tau}(A)(y)=\bigwedge_{x \in X}(A(x) \rightarrow$ $\left.\mathcal{K}_{\tau}\left(\top_{x}\right)(y)\right)$. Since $\tau=\left\{\mathcal{K}_{\tau}(A) \mid A \in L^{X}\right\}$, we have

$$
\begin{aligned}
e_{\tau}(x, y) & =\bigwedge_{A \in \tau}(A(x) \rightarrow A(y))=\bigwedge_{A \in L^{X}}\left(\mathcal{K}_{\tau}(A)(x) \rightarrow \mathcal{K}_{\tau}(A)(y)\right) \\
& \leq \bigwedge_{x \in X}\left(\mathcal{K}_{\tau}\left(\top_{z}\right)(x) \rightarrow \mathcal{K}_{\tau}\left(\top_{z}\right)(y)\right) . \\
e_{\tau}(x, y) & =\bigwedge_{A \in \tau}(A(x) \rightarrow A(y))=\bigwedge_{A \in L^{X}}\left(\mathcal{K}_{\tau}(A)(x) \rightarrow \mathcal{K}_{\tau}(A)(y)\right) \\
& =\bigwedge_{A \in L^{X}}\left(\bigwedge _ { z \in X } \left(A(z) \rightarrow \mathcal{K}_{\tau}\left(\top_{z}\right)(x) \rightarrow \bigwedge_{w \in X}\left(A(w) \rightarrow \mathcal{K}_{\tau}\left(\top_{w}\right)(y)\right)\right.\right. \\
& =\bigwedge_{A \in L^{X}} \bigwedge_{z \in X}\left(\left(A(z) \rightarrow \mathcal{K}_{\tau}\left(\top_{z}\right)(x)\right) \rightarrow\left(A(z) \rightarrow \mathcal{K}_{\tau}\left(\top_{z}\right)(y)\right)\right. \\
& \geq \bigwedge_{x \in X}\left(\mathcal{K}_{\tau}\left(\top_{z}\right)(x) \rightarrow \mathcal{K}_{\tau}\left(\top_{z}\right)(y)\right) .
\end{aligned}
$$

(10) Since $A=\bigvee_{x \in X}\left(A(x) \odot \top_{x}\right)$, by (4) and (5), $\mathcal{K}_{\tau}(A)(y)=\bigwedge_{x \in X}(A(x) \rightarrow$ $\left.\mathcal{K}_{\tau}\left(\top_{x}\right)(y)\right)=\bigwedge_{x \in X}\left(\mathcal{K}_{\tau}^{*}\left(\top_{x}\right)(y) \rightarrow A^{*}(x)\right)$. Put $e_{K_{\tau}}(x, y)=\mathcal{K}_{\tau}^{*}\left(\top_{x}\right)(y)$. Then

$$
\begin{gathered}
\mathcal{K}_{\tau}(A)(y)=\bigwedge_{x \in X}\left(e_{K_{\tau}}(x, y) \rightarrow A^{*}(x)\right) \\
e_{K_{\tau}}(x, x)=\mathcal{K}_{\tau}^{*}\left(\top_{x}\right)(x) \geq \top_{x}(x)=\top \\
\bigvee_{y \in X}\left(e_{K_{\tau}}(x, y) \odot e_{K_{\tau}}(y, z)\right) \leq e_{K_{\tau}}(x, z) \\
\text { iff } \bigvee_{y \in X}\left(\mathcal{K}_{\tau}^{*}\left(\top_{x}\right)(y) \odot \mathcal{K}_{\tau}^{*}\left(\top_{y}\right)(z)\right) \leq \mathcal{K}_{\tau}^{*}\left(\top_{x}\right)(z) \\
\text { iff } \bigwedge_{y \in X}\left(\mathcal{K}_{\tau}^{*}\left(\top_{x}\right)(y) \rightarrow \mathcal{K}_{\tau}\left(\top_{y}\right)(z)\right) \geq \mathcal{K}_{\tau}\left(\top_{x}\right)(z) \\
\text { iff } \mathcal{K}_{\tau}\left(\bigvee_{y \in X}\left(\mathcal{K}_{\tau}^{*}\left(\top_{x}\right)(y) \odot \top_{y}\right)\right)(z) \geq \mathcal{K}_{\tau}\left(\top_{x}\right)(z) \\
\text { iff } \mathcal{K}_{\tau}\left(\mathcal{K}_{\tau}^{*}\left(\top_{x}\right)\right)(z) \geq \mathcal{K}_{\tau}\left(\top_{x}\right)(z) .
\end{gathered}
$$

Hence $e_{K_{\tau}}$ is a fuzzy preorder.

By (9),

$$
\begin{aligned}
e_{\tau}(x, y) & =\bigwedge_{z \in X}\left(\mathcal{K}_{\tau}\left(\top_{z}\right)(x) \rightarrow \mathcal{K}_{\tau}\left(\top_{z}\right)(y)\right. \\
& =\bigwedge_{z \in X}\left(\mathcal{K}_{\tau}^{*}\left(\top_{z}\right)(y) \rightarrow \mathcal{K}_{\tau}^{*}\left(\top_{z}\right)(x)\right. \\
& =\bigwedge_{z \in X}\left(e_{K_{\tau}}(z, y) \rightarrow e_{K_{\tau}}(z, x)\right) \\
& \leq e_{K_{\tau}}(y, y) \rightarrow e_{K_{\tau}}(y, x)=e_{K_{\tau}}(y, x)
\end{aligned}
$$

Since $e_{K_{\tau}}$ is a fuzzy preorder, $e_{K_{\tau}}(z, y) \odot e_{K_{\tau}}(y, x) \leq e_{K_{\tau}}(z, x)$. Thus

$$
e_{K_{\tau}}(y, x) \leq \bigwedge_{z \in X}\left(e_{K_{\tau}}(z, y) \rightarrow e_{K_{\tau}}(z, x)\right)=e_{\tau}(x, y) .
$$

Hence $e_{K_{\tau}}(y, x)=e_{\tau}(y, x)$. 
Theorem 3.2. Let $\tau$ be an Alexandrov topology on $X$. Define $\mathcal{M}_{\tau}: L^{X} \rightarrow$ $L^{X}$ as follows:

$$
\mathcal{M}_{\tau}(A)=\bigwedge_{B \in \tau}\left(e_{L^{X}}\left(A^{*}, B\right) \rightarrow B\right) .
$$

Then the following properties hold.

(1) $e_{L^{X}}(A, B) \leq e_{L^{X}}\left(\mathcal{M}_{\tau}(B), \mathcal{M}_{\tau}(A)\right)$, for all $A, B \in L^{X}$.

(2) $A^{*} \leq \mathcal{M}_{\tau}(A)$ for all $A \in L^{X}$.

(3) $\mathcal{M}_{\tau}\left(\mathcal{M}_{\tau}^{*}(A)\right)=\mathcal{M}_{\tau}(A)$ for all $A \in L^{X}$.

(4) $\mathcal{M}_{\tau}(\alpha \rightarrow A)=\alpha \odot \mathcal{M}_{\tau}(A)$ for all $\alpha \in L, A \in L^{X}$.

(5) $\mathcal{M}_{\tau}\left(\bigwedge_{i \in \Gamma} A_{i}\right)=\bigvee_{i \in \Gamma} \mathcal{M}_{\tau}\left(A_{i}\right)$ for all $A_{i} \in L^{X}$.

(6) $\mathcal{M}_{\tau}(\sqcap \Phi)=\sqcup \mathcal{M}_{\tau}(\Phi)$ for each $\Phi: L^{X} \rightarrow L$ where $\mathcal{M}_{\tau} \rightarrow: L^{L^{X}} \rightarrow L^{L^{X}}$ defined as $\mathcal{M}_{\tau}^{\rightarrow}(\Phi)(B)=\bigvee_{\mathcal{M}_{\tau}(A)=B}(\Phi(A))$.

(7) $\mathcal{M}_{\tau}(A)=\bigwedge\left\{B \in L^{X} \mid A^{*} \leq B, B \in \tau\right\}$. $\left.L^{X}\right\}$.

(8) Define $\tau_{\mathcal{M}_{\tau}}=\left\{A \mid A=\mathcal{M}_{\tau}\left(A^{*}\right)\right\}$. Then $\tau=\tau_{\mathcal{M}_{\tau}}=\left\{\mathcal{M}_{\tau}\left(A^{*}\right) \mid A \in\right.$

(9) Define $e_{\tau}: X \times X \rightarrow L$ as follows

$$
e_{\tau}(x, y)=\bigwedge_{A \in \tau}(A(x) \rightarrow A(y)) .
$$

Then $e_{\tau}$ is a fuzzy preorder such that

$$
e_{\tau}(x, y)=\bigwedge_{A \in \tau}\left(\mathcal{M}_{\tau}\left(\top_{z}^{*}\right)(x) \rightarrow \mathcal{M}_{\tau}\left(\top_{z}^{*}\right)(y)\right) .
$$

Moreover, $e_{\tau^{*}}(x, y)=e_{\tau}(y, x)=e_{\tau}^{-1}(x, y)$.

(10) $\left(\mathcal{M}_{\tau}\left(A^{*}\right)\right)^{*}=\mathcal{K}_{\tau^{*}}(A)$ for all $A \in L^{X}$.

(11) There exists a fuzzy preorder $e_{M_{\tau}}: X \times X \rightarrow L$ such that

$$
\begin{aligned}
& \mathcal{M}_{\tau}(A)(y)=\bigvee_{x \in X}\left(e_{M_{\tau}}(x, y) \odot A^{*}(x)\right), \\
& \mathcal{K}_{\tau^{*}}(A)(y)=\bigwedge_{x \in X}\left(e_{M_{\tau}}(x, y) \rightarrow A^{*}(x)\right) .
\end{aligned}
$$

Moreover, $e_{M_{\tau}}(x, y)=e_{K_{\tau}}(y, x)=e_{\tau}(x, y)=e_{M_{\tau^{*}}}(y, x)=e_{K_{\tau}^{*}}(x, y)=e_{\tau^{*}}(y, x)$ for all $x, y \in X$.

Proof. (1) By Lemma 2.2 (6,8), we have

$$
\begin{aligned}
& e_{L^{X}}\left(\mathcal{M}_{\tau}(B), \mathcal{M}_{\tau}(A)\right) \\
& =\bigwedge_{x \in X}\left(\bigwedge_{C \in \tau}\left(e_{L^{X}}\left(B^{*}, C\right) \rightarrow C(x)\right) \rightarrow \bigwedge_{D \in \tau}\left(e_{L^{X}}\left(A^{*}, D\right) \rightarrow D(x)\right)\right. \\
& \geq \bigwedge_{x \in X} \bigwedge_{C \in \tau}\left(\left(e_{L^{X}}\left(B^{*}, C\right) \rightarrow C(x)\right) \rightarrow\left(e_{L^{X}}\left(A^{*}, C\right) \rightarrow C(x)\right)\right. \\
& \geq \bigwedge_{C \in \tau}\left(\left(e_{L^{X}}\left(A^{*}, C\right) \rightarrow\left(e_{L^{X}}\left(B^{*}, C\right)\right)\right.\right. \\
& \geq e_{L^{X}}\left(B^{*}, A^{*}\right)=e_{L^{X}}(A, B) .
\end{aligned}
$$


(2) Since $e_{L^{X}}\left(A^{*}, B\right) \odot A^{*} \leq B$ iff $A^{*} \leq e_{L^{X}}\left(A^{*}, B\right) \rightarrow B$, then $A^{*} \leq$ $\mathcal{M}_{\tau}(A)$.

(3) Since $\mathcal{M}_{\tau}(A) \in \tau$, then $\mathcal{M}_{\tau}\left(\mathcal{M}_{\tau}^{*}(A)\right) \leq e_{L^{X}}\left(\mathcal{M}_{\tau}(A), \mathcal{M}_{\tau}(A)\right) \rightarrow \mathcal{M}_{\tau}(A)=$ $\mathcal{M}_{\tau}(A)$. By $(2), \mathcal{M}_{\tau}\left(\mathcal{M}_{\tau}^{*}(A)\right)=\mathcal{M}_{\tau}(A)$.

(4) Since $\alpha \odot A^{*} \leq \alpha \odot \mathcal{M}_{\tau}(A)$ and $\alpha \odot \mathcal{M}_{\tau}(A) \in \tau$,

$$
\begin{aligned}
\mathcal{M}_{\tau}(\alpha \rightarrow A) & \leq e_{L^{X}}\left(\alpha \odot A^{*}, \alpha \odot \mathcal{M}_{\tau}(A)\right) \rightarrow \alpha \odot \mathcal{M}_{\tau}(A) \\
& =\alpha \odot \mathcal{M}_{\tau}(A) .
\end{aligned}
$$

Thus, $\mathcal{M}_{\tau}(\alpha \rightarrow A)=\alpha \odot \mathcal{M}_{\tau}(A)$.

(5) By (1), since $\mathcal{M}_{\tau}(B) \leq \mathcal{M}_{\tau}(A)$ for $A \leq B, \bigvee_{i \in \Gamma} \mathcal{M}_{\tau}\left(A_{i}\right) \leq \mathcal{M}_{\tau}\left(\bigwedge_{i \in \Gamma} A_{i}\right)$. Since $\bigvee_{i \in \Gamma} A_{i}^{*} \leq \bigvee_{i \in \Gamma} \mathcal{M}_{\tau}\left(A_{i}\right)$ and $\bigvee_{i \in \Gamma} \mathcal{M}_{\tau}\left(A_{i}\right) \in \tau$, we have

$$
\begin{aligned}
\mathcal{M}_{\tau}\left(\bigwedge_{i \in \Gamma} A_{i}\right) & \leq e_{L^{X}}\left(\bigvee_{i \in \Gamma} A_{i}^{*}, \bigvee_{i \in \Gamma} \mathcal{M}_{\tau}\left(A_{i}\right)\right) \rightarrow \bigvee_{i \in \Gamma} \mathcal{M}_{\tau}\left(A_{i}\right) \\
& =\bigvee_{i \in \Gamma} \mathcal{M}_{\tau}\left(A_{i}\right) .
\end{aligned}
$$

Hence $\mathcal{M}_{\tau}\left(\bigwedge_{i \in \Gamma} A_{i}\right)=\bigvee_{i \in \Gamma} \mathcal{M}_{\tau}\left(A_{i}\right)$.

(6) For each $\Phi: L^{X} \rightarrow L$, put $P=\sqcap \mathcal{M}_{\tau}^{\rightarrow}(\Phi)$. Since $\mathcal{M}_{\tau} \rightarrow(\Phi): L^{X} \rightarrow L$ is a map, we have $\sqcup \mathcal{M}_{\tau}(\Phi)=\bigvee_{C \in \tau}\left(\mathcal{M}_{\tau}(\Phi)(C) \odot C\right)$ and $P=\sqcup \mathcal{M}_{\tau} \rightarrow(\Phi)=$ $\mathcal{M}_{\tau}(\sqcup \Phi)$ from:

$$
\begin{aligned}
e_{L^{X}}(P, B) & =\bigwedge_{C \in L^{X}}\left(\mathcal{M}_{\tau} \rightarrow(\Phi)(C) \rightarrow e_{L^{X}}(C, B)\right. \\
& =\bigwedge_{C \in L^{X}} e_{L^{X}}\left(\mathcal{M}_{\tau}^{\rightarrow}(\Phi)(C) \odot C, B\right) \\
& =e_{L^{X}}\left(\bigvee_{C \in L^{X}}\left(\mathcal{M}_{\tau}(\Phi)(C) \odot C\right), B\right) \\
& =e_{L^{X}}\left(\bigvee_{A \in L^{X}}\left(\Phi(A) \odot \mathcal{M}_{\tau}(A)\right), B\right) \\
& =e_{L^{X}}\left(\mathcal{M}_{\tau}\left(\bigwedge_{A \in L^{X}}(\Phi(A) \rightarrow A)\right), B\right) \quad(\text { by }(4) \text { and }(5)) \\
& =e_{L^{X}}\left(\mathcal{M}_{\tau}(\sqcap \Phi), B\right)
\end{aligned}
$$

(7) Put $M(A)=\bigwedge\left\{B \in L^{X} \mid A^{*} \leq B, B \in \tau\right\}$. Since $A^{*} \leq M(A)$ and $M(A) \in \tau$, we have

$$
\mathcal{M}_{\tau}(A)=\bigwedge_{B \in \tau}\left(e_{L^{X}}\left(A^{*}, B\right) \rightarrow B\right) \leq e_{L^{X}}\left(A^{*}, M(A)\right) \rightarrow M(A)=M(A) .
$$

Since $A^{*} \leq \mathcal{M}_{\tau}(A)$ and $\mathcal{M}_{\tau}(A) \in \tau$, we have $M(A) \leq \mathcal{M}_{\tau}(A)$. Hence $\mathcal{M}_{\tau}=M$.

(8) We have $\tau=\tau_{\mathcal{M}_{\tau}}$ from $A \in \tau$ iff $\mathcal{M}_{\tau}\left(A^{*}\right)=A$ iff $A \in \tau_{\mathcal{M}_{\tau}}$. Put $\tau=\left\{\mathcal{M}_{\tau}\left(A^{*}\right) \mid A \in L^{X}\right\}$. Let $A \in \tau$. Then $A=\mathcal{M}_{\tau}\left(A^{*}\right)$. Let $\mathcal{M}_{\tau}\left(A^{*}\right) \in \tau_{1}$. Then $\mathcal{M}_{\tau}\left(\mathcal{M}_{\tau}^{*}\left(A^{*}\right)\right)=\mathcal{M}_{\tau}\left(A^{*}\right) \in \tau_{\mathcal{M}_{\tau}}=\tau$. Hence $\tau=\tau_{1}$. 
(9) By (8), since $\mathcal{M}_{\tau}\left(\top_{z}^{*}\right) \in \tau$,

$$
e_{\tau}(x, y)=\bigwedge_{A \in \tau}(A(x) \rightarrow A(y)) \leq \bigwedge_{x \in X}\left(\mathcal{M}_{\tau}\left(\top_{z}^{*}\right)(x) \rightarrow \mathcal{M}_{\tau}\left(\top_{z}^{*}\right)(y)\right) .
$$

Since $A^{*}=\bigwedge_{z \in X}\left(A(z) \rightarrow \top_{z}^{*}\right)$, by (4) and $(5), \mathcal{M}_{\tau}\left(A^{*}\right)(x)=\bigvee_{z \in X}(A(z) \odot$ $\left.\mathcal{M}_{\tau}\left(\top_{z}^{*}\right)(x)\right)$.

$$
\begin{aligned}
e_{\tau}(x, y) & =\bigwedge_{A \in \tau}(A(x) \rightarrow A(y))=\bigwedge_{A \in L^{X}}\left(\mathcal{M}_{\tau}\left(A^{*}\right)(x) \rightarrow \mathcal{M}_{\tau}\left(A^{*}\right)(y)\right) \\
& =\bigwedge_{A \in L^{X}}\left(\bigwedge _ { z \in X } \left(A(z) \odot \mathcal{M}_{\tau}\left(\top_{z}^{*}\right)(x) \rightarrow \bigwedge_{w \in X}\left(A(w) \odot \mathcal{M}_{\tau}\left(\top_{w}^{*}\right)(y)\right)\right.\right. \\
& =\bigwedge_{A \in L^{X}} \bigwedge_{z \in X}\left(\left(A(z) \odot \mathcal{M}_{\tau}\left(\top_{z}^{*}\right)(x)\right) \rightarrow\left(A(z) \odot \mathcal{M}_{\tau}\left(\top_{z}^{*}\right)(y)\right)\right. \\
& \geq \bigwedge_{x \in X}\left(\mathcal{M}_{\tau}\left(\top_{z}^{*}\right)(x) \rightarrow \mathcal{M}_{\tau}\left(\top_{z}^{*}\right)(y)\right) .
\end{aligned}
$$

(10)

$$
\begin{aligned}
\left(\mathcal{M}_{\tau}\left(A^{*}\right)\right)^{*} & =\left(\bigwedge_{B \in \tau}\left(e_{L^{X}}(A, B) \rightarrow B\right)\right)^{*} \\
& =\bigvee_{B \in \tau}\left(e_{L^{X}}\left(B^{*}, A^{*}\right) \odot B^{*}\right) \\
& =\bigvee_{B^{*} \in \tau^{*}}\left(e_{L^{X}}\left(B^{*}, A^{*}\right) \odot B^{*}\right) \\
& =\mathcal{K}_{\tau^{*}}(A) .
\end{aligned}
$$

(11) Since $A=\bigwedge_{z \in X}\left(A^{*}(z) \rightarrow \top_{z}^{*}\right)$, by (4) and (5),

$$
\mathcal{M}_{\tau}(A)(x)=\mathcal{M}_{\tau}\left(\bigwedge_{z \in X}\left(A^{*}(z) \rightarrow \top_{z}^{*}\right)\right)(x)=\bigvee_{z \in X}\left(A^{*}(z) \odot \mathcal{M}_{\tau}\left(\top_{z}^{*}\right)(x)\right) .
$$

Put $e_{M_{\tau}}(x, y)=\mathcal{M}_{\tau}\left(\top_{x}^{*}\right)(y)$. Then

$$
\begin{gathered}
\mathcal{M}_{\tau}(A)(y)=\bigvee_{x \in X}\left(e_{M_{\tau}}(x, y) \odot A^{*}(x)\right) \\
e_{M_{\tau}}(x, x)=\mathcal{M}_{\tau}\left(\top_{x}^{*}\right)(x) \geq \top_{x}(x)=\top \\
\bigvee_{y \in X}\left(e_{M_{\tau}}(x, y) \odot e_{M_{\tau}}(y, z)\right) \leq e_{M_{\tau}}(x, z) \\
\text { iff } \bigvee_{y \in X}\left(\mathcal{M}_{\tau}\left(\top_{x}^{*}\right)(y) \odot \mathcal{M}_{\tau}\left(\top_{y}^{*}\right)(z)\right) \leq \mathcal{M}_{\tau}\left(\top_{x}^{*}\right)(z) \\
\text { iff } \mathcal{M}_{\tau}\left(\bigwedge_{y \in X}\left(\mathcal{M}_{\tau}\left(\top_{x}^{*}\right)(y) \rightarrow \top_{y}^{*}\right)\right)(z) \leq \mathcal{M}_{\tau}\left(\top_{x}^{*}\right)(z) \\
\text { iff } \mathcal{M}_{\tau}\left(\mathcal{M}_{\tau}^{*}\left(\top_{x}^{*}\right)\right)(z) \leq \mathcal{M}_{\tau}\left(\top_{x}^{*}\right)(z)
\end{gathered}
$$

Hence $e_{M_{\tau}}$ is a fuzzy preorder. Since $e_{M_{\tau}}(x, y)=\mathcal{M}_{\tau}\left(\top_{x}^{*}\right)(y)=\mathcal{K}_{\tau^{*}}^{*}\left(\top_{x}\right)(y)$, by Theorem 3.1(10),

$$
\mathcal{K}_{\tau^{*}}(A)(y)=\bigwedge_{x \in X}\left(e_{M_{\tau}}(x, y) \rightarrow A^{*}(x)\right) .
$$

Moreover, by Theorem 3.1(10), $e_{M_{\tau}}(x, y)=e_{K_{\tau}}(y, x)=e_{\tau}(x, y)=e_{M_{\tau^{*}}}(y, x)=$ $e_{K_{\tau}^{*}}(x, y)=e_{\tau^{*}}(y, x)$ for all $x, y \in X$. 
Example 3.3.Let $\left(L=[0,1], \odot, \rightarrow{ }^{*}\right)$ be a complete residuated lattice with a strong negation defined by

$$
x \odot y=(x+y-1) \vee 0, x \rightarrow y=(1-x+y) \wedge 1, x^{*}=1-x .
$$

Let $X=\{x, y, z\}$ be a set and $B \in L^{X}$ as follows:

$$
B(x)=0.5, B(y)=0.2, B(z)=0.9 .
$$

Define $e_{B}(x, y)=B(x) \rightarrow B(y)$ such that

$$
e_{B}=\left(\begin{array}{ccc}
1 & 0.7 & 1 \\
1 & 1 & 1 \\
0.6 & 0.3 & 1
\end{array}\right)
$$

(1) We define

$$
\tau=\left\{e_{B}(A)(y)=\bigvee_{y \in X}\left(e_{B}(x, y) \odot A(x)\right) \mid A \in L^{X}\right\} .
$$

(T1) For $\perp_{X} \in L^{X}, e_{B}\left(\perp_{X}\right)=\perp_{X} \in \tau$. For $\top_{X} \in L^{X}, e_{B}\left(\top_{X}\right)=\top_{X} \in \tau$.

(T2) For $e_{B}\left(A_{i}\right) \in \tau$ for each $i \in \Gamma, \bigvee_{i \in \Gamma} e_{B}\left(A_{i}\right)=e_{B}\left(\bigvee_{i \in \Gamma} A_{i}\right) \in \tau$. Moreover, since $e_{B}(A)(x) \geq e_{B}(x, x) \odot A(x)=A(x)$ and $e_{B}\left(e_{X}(A)\right)=e_{X}(A)$,

$$
\bigwedge_{i \in \Gamma} e_{B}\left(A_{i}\right) \leq e_{B}\left(\bigwedge_{i \in \Gamma} e_{B}\left(A_{i}\right)\right) \leq \bigwedge_{i \in \Gamma} e_{B}\left(e_{B}\left(A_{i}\right)\right)=\bigwedge_{i \in \Gamma} e_{B}\left(A_{i}\right) .
$$

Hence $\bigwedge_{i \in \Gamma} e_{B}\left(A_{i}\right)=e_{B}\left(\bigwedge_{i \in \Gamma} A_{i}\right) \in \tau$.

(T3) For $e_{B}(A) \in \tau, \alpha \odot e_{B}(A)=e_{B}(\alpha \odot A) \in \tau$.

(T4) Since $\alpha \odot e_{B}\left(\alpha \rightarrow e_{B}(A)\right) \leq e_{B}\left(e_{B}(A)\right)=e_{B}(A)$, we have

$$
\alpha \rightarrow e_{B}(A) \leq e_{B}\left(\alpha \rightarrow e_{B}(A)\right) \leq \alpha \rightarrow e_{B}(A)
$$

Hence, for $e_{B}(A) \in \tau, \alpha \rightarrow e_{B}(A)=e_{B}\left(\alpha \rightarrow e_{B}(A)\right) \in \tau$. Hence $\tau$ is an Alexandrov topology on $X$.

(2) Since $B(x) \rightarrow B(y) \leq(\alpha \odot B)(x) \rightarrow(\alpha \odot B)(y), B(x) \rightarrow B(y) \leq$ $(\alpha \rightarrow B)(x) \rightarrow(\alpha \rightarrow B)(y)$, we have $e_{\tau}(x, y)=e_{B}(x, y)$. Moreover, $e_{\tau^{*}}(x, y)=$ $e_{B^{*}}(x, y)=e_{B}(y, x)$.

Since

$$
\tau=\left\{(x \odot(1,0.7,1)) \vee(y \odot(1,1,1)) \vee(z \odot(0.6,0.3,1)) \mid(x, y, z) \in L^{X}\right\},
$$


by Theorem 3.1 (7), we obtain:

$$
\begin{aligned}
\mathcal{K}_{\tau}\left(1_{x}\right)= & \bigvee\{A \in \tau \mid A=(x \odot(1,0.7,1)) \vee(y \odot(1,1,1)) \\
& \vee(z \odot(0.6,0.3,1)) \leq(0,1,1)\}=(0,0,0.4) \\
\mathcal{K}_{\tau}\left(1_{y}\right)= & \bigvee\{A \in \tau \mid A=(x \odot(1,0.7,1)) \vee(y \odot(1,1,1)) \\
& \vee(z \odot(0.6,0.3,1)) \leq(1,0,1)\}=(0.3,0,0.7) \\
\mathcal{K}_{\tau}\left(1_{z}\right)= & \bigvee\{A \in \tau \mid A=(x \odot(1,0.7,1)) \vee(y \odot(1,1,1)) \\
& \vee(z \odot(0.6,0.3,1)) \leq(1,1,0)\}=(0,0,0)
\end{aligned}
$$

Put $e_{K_{\tau}}(x, y)=\mathcal{K}_{\tau}^{*}\left(1_{x}\right)(y)$, then

$$
e_{K_{\tau}}(x, y)=\mathcal{K}_{\tau}^{*}\left(1_{x}\right)(y)=e_{\tau}(y, x)=e_{B}(y, x)=e_{\tau^{*}}(x, y) .
$$

(3) By Theorem $3.2(7)$, we obtain:

$$
\begin{aligned}
& \mathcal{M}_{\tau}\left(1_{x}^{*}\right)= \bigwedge\{A \in \tau \mid(1,0,0) \leq A=(x \odot(1,0.7,1)) \vee(y \odot(1,1,1)) \\
&\vee(z \odot(0.6,0.3,1))\}=(1,0.7,1) \\
& \mathcal{M}_{\tau}\left(1_{y}^{*}\right)=\bigwedge\{A \in \tau \mid(0,1,0) \leq A=(x \odot(1,0.7,1)) \vee(y \odot(1,1,1)) \\
&\vee \vee(z \odot(0.6,0.3,1))\}=(1,1,1) \\
& \mathcal{M}_{\tau}\left(1_{z}^{*}\right)=\bigwedge\{A \in \tau \mid(0,0,1) \leq A=(x \odot(1,0.7,1)) \vee(y \odot(1,1,1)) \\
&\vee(z \odot(0.6,0.3,1))\}=(0.6,0.3,1)
\end{aligned}
$$

Moreover, we have $e_{M_{\tau}}(y, x)=\mathcal{M}_{\tau}\left(1_{y}^{*}\right)(x)=e_{K_{\tau}}(x, y)=\mathcal{K}_{\tau}^{*}\left(1_{x}\right)(y)=e_{\tau}(y, x)=$ $e_{B}(y, x)=e_{\tau^{*}}(x, y)$.

(4) For $D_{1}=(0.5,0.3,0.7), D_{2}=(0.4,0.8,0.5)$, we obtain

$$
\begin{gathered}
\mathcal{K}_{\tau}\left(D_{i}\right)(y)=\bigwedge_{x \in X}\left(e_{K_{\tau}}(x, y) \rightarrow D_{i}^{*}(x)\right)=\bigwedge_{x \in X}\left(e_{B}(y, x) \rightarrow D_{i}^{*}(x)\right) \\
\mathcal{M}_{\tau}\left(D_{i}\right)(y)=\bigvee_{x \in X}\left(e_{M_{\tau}}(x, y) \odot D_{i}^{*}(x)\right)=\bigvee_{x \in X}\left(e_{B}(x, y) \odot D_{i}^{*}(x)\right) \\
\mathcal{K}_{\tau}\left(D_{1}\right)=(0.3,0.3,0.3), \mathcal{K}_{\tau}\left(D_{2}\right)=(0.5,0.2,0.5) \\
\mathcal{M}_{\tau}\left(D_{1}\right)=(0.7,0.7,0.7), \mathcal{M}_{\tau}\left(D_{2}\right)=(0.6,0.3,0.6)
\end{gathered}
$$

Let $\Phi: L^{X} \rightarrow L$ as follows

$$
\Phi(B)= \begin{cases}0.7, & \text { if } B=B_{1}, \\ 0.8, & \text { if } B=B_{2}, \\ 0, & \text { otherwise. }\end{cases}
$$

$\sqcap \Phi=\bigwedge_{A \in L^{X}}(\Phi(A) \rightarrow A)=\left(\Phi\left(D_{1}\right) \rightarrow D_{1}\right) \wedge\left(\Phi\left(D_{2}\right) \rightarrow D_{2}\right)$ $=(0.7 \rightarrow(0.5,0.3,0.7)) \wedge(0.8 \rightarrow(0.4,0.8,0.5))=(0.6,0.6,0.7)$ 


$$
\begin{aligned}
& \sqcup \Phi= \bigvee_{A \in L^{X}}(\Phi(A) \odot A)=\left(\Phi\left(D_{1}\right) \odot D_{1}\right) \vee\left(\Phi\left(D_{2}\right) \odot D_{2}\right) \\
&=(0.7 \odot(0.5,0.3,0.7)) \vee(0.8 \odot(0.4,0.8,0.5))=(0.2,0.6,0.4) \\
& \mathcal{K}_{\tau}(\sqcup \Phi)=(0.6,0.4,0.6) . \\
&\left.\sqcap \mathcal{K}_{\tau} \rightarrow \Phi\right)=\bigwedge_{A \in L^{X}}\left(\Phi(A) \rightarrow \mathcal{K}_{\tau}(A)\right) \\
&=\left(\Phi\left(D_{1}\right) \rightarrow \mathcal{K}_{\tau}\left(D_{1}\right)\right) \wedge\left(\Phi\left(D_{2}\right) \rightarrow \mathcal{K}_{\tau}\left(D_{2}\right)\right) \\
&=(0.7 \rightarrow(0.3,0.3,0.3)) \wedge(0.8 \rightarrow(0.5,0.2,0.5)) \\
&=(0.6,0.4,0.6)
\end{aligned}
$$

we have $\left.\mathcal{K}_{\tau}(\sqcup \Phi)=\sqcap \mathcal{K}_{\tau} \rightarrow \Phi\right)$.

$$
\begin{aligned}
& \mathcal{M}_{\tau}(\sqcap \Phi)=(0.4,0.4,0.4) \\
& \sqcup \mathcal{M}_{\tau}(\Phi)=\bigvee_{A \in L^{X}}\left(\Phi(A) \odot \mathcal{M}_{\tau}(A)\right) \\
& =\left(\Phi\left(D_{1}\right) \odot \mathcal{M}_{\tau}\left(D_{1}\right)\right) \vee\left(\Phi\left(D_{2}\right) \odot \mathcal{M}_{\tau}\left(D_{2}\right)\right) \\
& =(0.7 \odot(0.7,0.7,0.7)) \vee(0.8 \odot(0.6,0.3,0.6)) \\
& =(0.4,0.4,0.4)
\end{aligned}
$$

Thus, $\left.\mathcal{M}_{\tau}(\sqcap \Phi)=\sqcup \mathcal{M}_{\tau} \rightarrow \Phi\right)$.

(5) Since $\tau^{*}=\left\{A^{*} \mid A \in \tau\right\}$, we have

$$
\begin{aligned}
& \tau^{*}=\left\{\bigwedge_{x \in X}\left(e_{X}(x,-) \rightarrow B(x)\right) \mid B \in L^{X}\right\} . \\
& \mathcal{K}_{\tau^{*}}\left(1_{x}\right)= \bigwedge\left\{A \in \tau^{*} \mid A=((1,0.7,1) \rightarrow x) \wedge((1,1,1) \rightarrow y)\right. \\
&\wedge((0.6,0.3,1) \rightarrow z) \leq(0,1,1)\}=(0,0.3,0) \\
& \mathcal{K}_{\tau^{*}}\left(1_{y}\right)= \bigwedge\left\{A \in \tau^{*} \mid A=((1,0.7,1) \rightarrow x) \wedge((1,1,1) \rightarrow y)\right. \\
&\wedge((0.6,0.3,1) \rightarrow z) \leq(1,0,1)\}=(0,0,0) \\
& \mathcal{K}_{\tau^{*}}\left(1_{z}\right)= \bigwedge\left\{A \in \tau^{*} \mid A=((1,0.7,1) \rightarrow x) \wedge((1,1,1) \rightarrow y)\right. \\
&\wedge((0.6,0.3,1) \rightarrow z) \leq(1,1,0)\}=(0.4,0.7,0) \\
& \tau_{1}=\left\{e_{B^{*}}(A)(y)=\bigvee\left(e_{B^{*}}(x, y) \odot A(x)\right) \mid A \in L^{X}\right\} . \\
& y \in X \\
& \mathcal{K}_{\tau_{1}}\left(1_{x}\right)= \bigvee\left\{A \in \tau_{1} \mid A=(x \odot(1,1,0.6)) \vee(y \odot(0.7,1,0.3))\right. \\
&\vee(z \odot(1,1,1)) \leq(0,1,1)\}=(0,0.3,0) \\
& \mathcal{K}_{\tau_{1}}\left(1_{y}\right)= \bigvee\left\{A \in \tau_{1} \mid A=(x \odot(1,1,0.6)) \vee(y \odot(0.7,1,0.3))\right. \\
&\vee(z \odot(1,1,1)) \leq(1,0,1)\}=(0,0,0) \\
& \mathcal{K}_{\tau_{1}}\left(1_{z}\right)= \bigvee\left\{A \in \tau_{1} \mid A=(x \odot(1,1,0.6)) \vee(y \odot(0.7,1,0.3))\right. \\
&\vee(z \odot(1,1,1)) \leq(1,1,0)\}=(0.4,0.7,0)
\end{aligned}
$$

Hence $\mathcal{K}_{\tau_{1}}\left(1_{x}\right)(y)=\mathcal{K}_{\tau^{*}}\left(1_{x}\right)(y)$ for all $x, y \in X$, then $\mathcal{K}_{\tau_{1}}=\mathcal{K}_{\tau^{*}}$. Thus,

$$
\tau^{*}=\tau_{\mathcal{K}_{\tau^{*}}}^{*}=\tau_{\mathcal{K}_{\tau_{1}}}^{*}=\tau_{1}
$$


For $D_{1}, D_{2}$ and $\Phi$ in (3), we obtain

$$
\begin{gathered}
\mathcal{K}_{\tau^{*}}\left(D_{i}\right)(y)=\bigwedge_{x \in X}\left(e_{K_{\tau^{*}}}(x, y) \rightarrow D_{i}^{*}(x)\right) \\
\mathcal{M}_{\tau^{*}}\left(D_{i}\right)(y)=\bigvee_{x \in X}\left(e_{M_{\tau^{*}}}(x, y) \odot D_{i}^{*}(x)\right)=\bigvee_{x \in X}\left(e_{K_{\tau^{*}}}(y, x) \odot D_{i}^{*}(x)\right) . \\
\mathcal{K}_{\tau^{*}}\left(D_{1}\right)=(0.5,0.7,0.3), \mathcal{K}_{\tau^{*}}\left(D_{2}\right)=(0.2,0.2,0.2), \\
\mathcal{M}_{\tau}\left(D_{1}\right)=(0.5,0.7,0.3), \mathcal{M}_{\tau}\left(D_{2}\right)=(0.6,0.6,0.5)
\end{gathered}
$$

Since $\sqcup \Phi=(0.2,0.6,0.4)$ and

$$
\begin{aligned}
& \mathcal{K}_{\tau^{*}}(\sqcup \Phi)=(0.4,0.4,0.4) . \\
& \sqcap \mathcal{K}_{\tau^{*}}(\Phi)=\bigwedge_{A \in L^{X}}\left(\Phi(A) \rightarrow \mathcal{K}_{\tau^{*}}(A)\right) \\
& =\left(\Phi\left(D_{1}\right) \rightarrow \mathcal{K}_{\tau^{*}}\left(D_{1}\right)\right) \wedge\left(\Phi\left(D_{2}\right) \rightarrow \mathcal{K}_{\tau^{*}}\left(D_{2}\right)\right) \\
& =(0.7 \rightarrow(0.5,0.7,0.3)) \wedge(0.8 \rightarrow(0.2,0.2,0.2)) \\
& =(0.4,0.4,0.4)
\end{aligned}
$$

we have $\mathcal{K}_{\tau^{*}}(\sqcup \Phi)=\sqcap \mathcal{K}_{\tau^{*}}(\Phi)$.

Since $\sqcap \Phi=(0.6,0.6,0.7)$ and

$$
\begin{aligned}
& \mathcal{M}_{\tau^{*}}(\sqcap \Phi)=(0.4,0.4,0.3) \\
& \sqcup \mathcal{M}_{\tau^{*}}(\Phi)=\bigvee_{A \in L^{X}}\left(\Phi(A) \odot \mathcal{M}_{\tau^{*}}(A)\right) \\
& =\left(\Phi\left(D_{1}\right) \odot \mathcal{M}_{\tau^{*}}\left(D_{1}\right)\right) \vee\left(\Phi\left(D_{2}\right) \odot \mathcal{M}_{\tau^{*}}\left(D_{2}\right)\right) \\
& =(0.7 \odot(0.5,0.7,0.3)) \vee(0.8 \odot(0.6,0.6,0.5)) \\
& =(0.4,0.4,0.3),
\end{aligned}
$$

then $\mathcal{M}_{\tau^{*}}(\sqcap \Phi)=\sqcup \mathcal{M}_{\tau^{*}}^{\rightarrow}(\Phi)$.

\section{References}

[1] R. Bělohlávek,Fuzzy Relational Systems, Kluwer Academic Publishers, New York , (2002), doi: 10.1007/978-1-4615-0633-1.

[2] P. Hájek, Metamathematices of Fuzzy Logic, Kluwer Academic Publishers, Dordrecht (1998), doi: 10.1007/978-94-011-5300-3.

[3] Fang Jinming, I-fuzzy Alexandrov topologies and specialization orders, Fuzzy Sets and Systems, 158(2007), 2359-2374, doi: 10.1016/j.fss.2007.05.001. 
[4] Y.C. Kim, Alexandrov L-topologies International Journal of Pure and Applied Mathematics, 93(2)(2014), 165-179, doi: 10.12732/ijpam.v93i2.2.

[5] Y.C. Kim, Alexandrov $L$-topologies and $L$-join meet approximation operators International Journal of Pure and Applied Mathematics, 91(1)(2014), 113-129, doi: 10.12732/ijpam.v91i1.12.

[6] J. Kortelainen, On relationships between modified sets, topological spaces and rough sets, Fuzzy Sets and Systems, 61(1994), 91-95, doi: 10.1016/0165-0114(94)90288-7.

[7] H. Lai, D. Zhang, Fuzzy preorder and fuzzy topology, Fuzzy Sets and Systems, 157 (2006), 1865-1885, doi: 10.1016/j.fss.2006.02.013.

[8] Z. Pawlak, Rough sets, Int. J. Comput. Inf. Sci., 11 (1982), 341-356, , doi: $10.1007 / \mathrm{BF} 01001956$.

[9] Z. Pawlak, Rough probability, Bull. Pol. Acad. Sci. Math., 32(1984), 607615.

[10] Y.H. She, G.J. Wang, An axiomatic approach of fuzzy rough sets based on residuated lattices, Computers and Mathematics with Applications, 58 (2009), 189-201, doi: 10.1016/j.camwa.2009.03.100.

[11] Q. Y. Zhang, L. Fan, Continuity in quantitive domains, Fuzzy Sets and Systems, 154 (2005), 118-131, doi: 10.1016/j.fss.2005.01.007.

[12] Q. Y. Zhang, W. X. Xie, Fuzzy complete lattices, Fuzzy Sets and Systems, 160(2009), 2275-2291, doi: 10.1016/j.fss.2008.12.001. 